\begin{tabular}{|l|l|l||}
\hline \multicolumn{2}{|c|}{ PublisherInfo } \\
\hline \hline PublisherName & $:$ & BioMed Central \\
\hline \hline PublisherLocation & $:$ & London \\
\hline \hline PublisherImprintName & $:$ & BioMed Central \\
\hline \hline
\end{tabular}

\title{
Primary chemotherapy with CEF-infu
}

\begin{tabular}{|l|l|l||}
\hline \multicolumn{2}{|c||}{ ArticleInfo } \\
\hline \hline ArticleID & $:$ & 3790 \\
\hline \hline ArticleDOI & $:$ & $10.1186 /$ bcr-2002-74150 \\
\hline \hline ArticleCitationID & $:$ & 74150 \\
\hline \hline ArticleSequenceNumber & $:$ & 13 \\
\hline \hline ArticleCategory & $:$ & Paper Report \\
\hline \hline ArticleFirstPage & $:$ & 1 \\
\hline \hline ArticleLastPage & $:$ & 3 \\
\hline \hline & & RegistrationDate $: 2002-1-8$ \\
& $:$ & Received \\
\hline ArticleHistory & Accepted 2002-1-8 \\
& $:$ 2002-1-9 \\
\hline \hline ArticleCopyright & $:$ & Biomed Central Ltd2002 \\
\hline \hline ArticleGrants & $:$ & \\
\hline \hline ArticleContext & $:$ & 1305844 \\
\hline \hline
\end{tabular}


Fatima Cardoso, ${ }^{\text {Aff1 }}$

Aff1 Jules Bordet Institute, Brussels, Belgium

\section{Keywords}

Breast cancer, CEF-infu, phase I, primary chemotherapy

\section{Context}

Neoadjuvant chemotherapy for large operable (LO) or locally advanced (LA) breast cancer (BC) has been shown to increase the rate of conserving breast surgery, without compromising overall survival. However, the optimal regimen remains unknown. Previous studies using infusional 5-FU (5-FU-infu) in association with epirubicin and cisplatin/carboplatin (ECisF) for $\mathrm{LO}$ and/or LABC have shown high response rates and good tolerability. The dose of epirubicin used in those studies was $50 \mathrm{mg} / \mathrm{m}^{2}, \mathrm{q} 3 \mathrm{w}$. As there is a well-known dose-response effect for anthracyclines, the aim of this study was to identify the maximum tolerated dose of epirubicin, when given in association with the other two drugs.

\section{Significant findings}

A total of 21 patients ( $11 \mathrm{LO}, 8 \mathrm{LABC})$ were treated, with a median of 5 cycles per patient. An interim analysis showed that grade 3 palmar-plantar erythrodysesthesia (PPE) occurred in 5/16 patients at the 3 dose levels. Accordingly, the protocol was amended to limit the duration of 5-FU-infu from 4 to 3 weeks. Dose limiting toxicities were grade 3 febrile neutropenia (FN), grade 3 mucositis, and grade 3 infection. Epirubicin $60 \mathrm{mg} / \mathrm{m}^{2}$, day $1 \& 8$, q4w was considered the recommended dose. Objective responses were seen in $75 \%(15 / 20)$ of evaluable patients.

\section{Comments}


This study demonstrates the feasibility of the combination of 5-FU-infu with an escalating dose of epirubicin. The rate of haematological toxicity and/or FN (13\% on dose level 3 ) was lower than the one seen with Canadian FEC (see Additional information) at epirubicin $120 \mathrm{mg} / \mathrm{m}^{2} /$ cycle without prophylactic antibiotics $(18.8 \%)$. However, PPE was a major toxicity, particularly with the 4 -week 5-FU-infu. The increase time exposure to epirubicin, due to the day 1 and 8 fractionation, is proposed as a possible explanation ( $31 \%$ vs $10 \%$ with other regimens using 5 -FU-infu). This fractionation concomitantly with 5-FU-infu could result in cutaneous accumulation of both dugs, leading to an increased rate of PPE. After the protocol amendment no grade 3 PPE was seen. Given the encouraging clinical activity of this regimen, a new study was recently activated with capecitabine replacing 5-FUinfu. Given the heterogeneous bioavailability and the toxicity profile (mainly PPE and diarrhoea) of capecitabine, infusional 5-FU is still a regimen to consider in the metastatic setting. Despite being a phase I study, it would be interesting to know the percentage of patients that underwent conserving surgery or mastectomy and the pathological responses obtained.

\section{Methods}

Phase I clinical trial, dose escalation

\section{Additional information}

Levine MN, Bramwell V, Pritchard K, Perrault D, Findlay B, Abu-Zahra H, Warr D, Arnold A, Skillings J: A pilot study of intensive cyclophosphamide, epirubicin and fluorouracil in patients with axillary node positive or locally advanced breast cancer. Eur J Cancer 1992, 29A:37-43.

\section{References}

1. Bonnefoi H, Biganzoli L, Cufer T, Mauriac L, Hamilton A, Schaefer P, Piccart M: An EORTC phase I study of epirubicin in combination with fixed doses of cyclophosphamide and infusional 5-FU (CEFinfu) as primary treatment of large operable or locally advanced/inflammatory breast cancer. Breast Cancer Res Treat. 2001, 70: 55-63.

This PDF file was created after publication. 